\title{
Fractions Lab goes east: learning and interaction with an Exploratory Learning Environment in China
}

\author{
Manolis Mavrikis ${ }^{1}$, Wayne Holmes ${ }^{2}$, Jingjing Zhang $^{3}$ and Ning $\mathrm{Ma}^{3}$ \\ ${ }^{1}$ UCL Knowledge Lab, University College London, UK \\ ${ }^{2}$ Institute of Educational Technology, Open University, UK \\ ${ }^{3}$ School of Educational Technology \& AICFE, Beijing Normal University, China \\ m.mavrikis@ucl.ac.uk, jingjing.zhang@bnu.edu.cn
}

\begin{abstract}
In a bid to better understand cultural differences and feed into the design of an exploratory learning environment for learning fractions in China, we conducted a study in three schools in Beijing. A mixed methods protocol was followed involving 186 children. In this paper, we report several results, including a paired t-test suggesting a significant difference between pre- and post-tests and effect sizes warranting further research. Beyond learning gains, we also report preliminary results from analysis of student interaction data that points to similarities as well as differences between UK and China. This is important because it helps us determine next steps in terms of the design, implementation and integration of the technology in the two contexts, and raises future hypothesis.
\end{abstract}

Keywords: Exploratory Learning Environments, cultural differences, gaming.

\section{$1 \quad$ Introduction}

While educational technologies can promote both learning and interconnectivity between people in widely different contexts, issues arise when differences between cultural identities are ignored. In particular, especially given the increasing globalisation of technology in learning, much of it driven by western corporations, research is needed to investigate the impacts of specific technologies in different contexts. This is important in the AIED field as limited research so far has looked into cultural differences and commonalities (e.g., [1]) yet the role of affect and feedback in different cultures is critical for the design that underpins AIED systems.

Our focus is on the UK and China, two educational systems that are literally a world apart [2]. Despite the emphasis on 'mastery learning' in both countries, the implementations differ widely [3], as do the results (the most recent PISA reports put the UK at 26 out of 40 countries in mathematics education, while Shanghai China tops the list (see http://www.oecd.org/pisa/). Nonetheless, given the importance placed on mathematics in both cultures, our case study is fractions, a core mathematics topic that can prove difficult for young students to master, with important implications later on. We are particularly interested in the role of digital multiple representations and intelligent feedback on the conceptual and procedural knowledge of fractions.

Accordingly, this paper presents work aimed at improving our understanding of the role of a particular digital environment on learning, known as Fractions Lab, which is a virtual manipulative with exploratory tasks and intelligent feedback. Our overarching 
aim was to investigate how Fractions Lab worked in China and what kind of differences and commonalities one can observe in its use. The current study builds upon earlier research in the UK and Germany [4], by investigating the use of Fractions Lab in three schools in Beijing involving 186 children (Fig. 1). In particular, beyond learning gains, we look at key issues such as the use of feedback, student perceived difficulty of the activities and gaming behaviours, comparing findings from the UK and China.

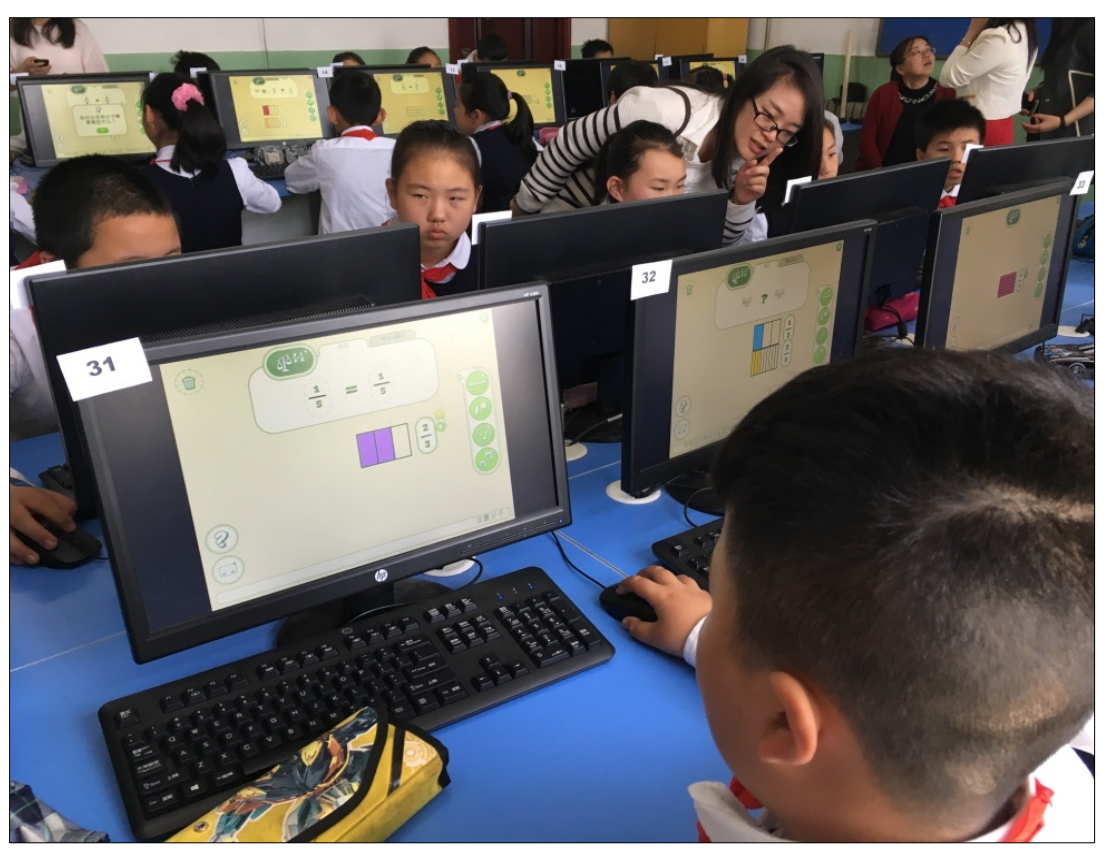

Fig. 1. Fractions Lab being used by students in a Beijing school.

\section{Fractions Lab}

Fractions Lab is an exploratory learning environment that enables interaction via direct manipulation [5]. It was developed as a component of the EU-funded iTalk2Learn project's intelligent tutoring platform (for details see $[6,7]$ ). While we have evidence from the UK and Germany about the overall efficacy of the platform [4, 7], the impact of cultural identity (even between two West European countries) is not well understood. This is especially true for exploratory learning environments and student interaction.

Fractions Lab, which is now a stand-alone programme (Fig. 2), aims to foster conceptual knowledge, which we define as implicit or explicit understanding about underlying principles and structures of a domain [8]. The focus of this type of knowledge lies in understanding why, for example, different mathematical principles refer to each other and on making sense of these connections. Conceptual understanding of equivalent fractions, for example, includes being able to make connections between fraction representations by understanding what is the same and what is different and by showing that a fraction represents a number [9].

A detailed description of the design decisions behind Fractions Lab can be found in [5]. Here it suffices to say that various tools enable a student to change a fraction representation's numerator and denominator, to partition a representation, or to copy it. 


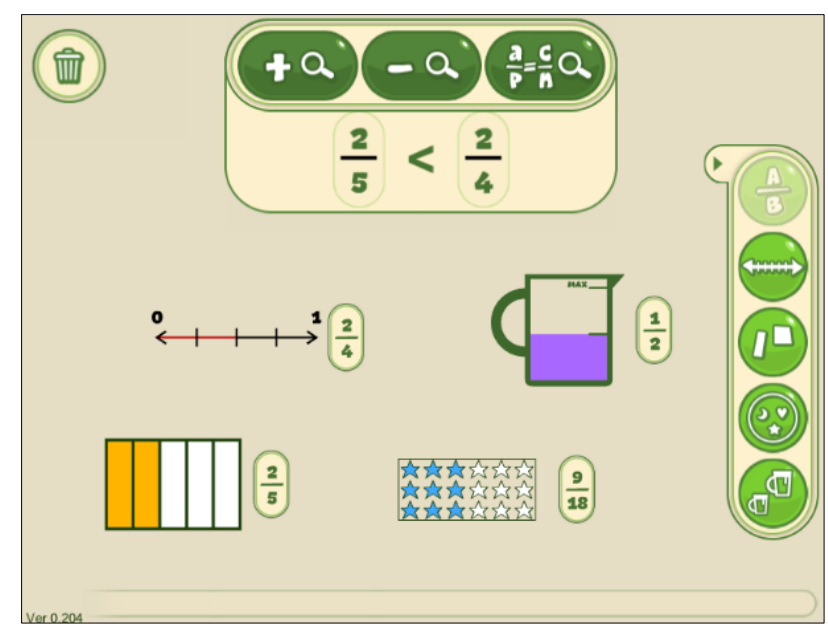

Fig. 2. Screenshot of Fractions Lab with various representations.

The addition, subtraction and comparison tools (at the top of the screen shown in Fig. 2) allow students to check their hypotheses and adopt a constructivist stance to learning. Corresponding tasks support students' conceptual development $[4,5]$.

\section{Research objectives and methodology}

The aim of our research was to explore both (i) how to support students' conceptual knowledge of fractions in China, and (ii) the cultural similarities and differences between UK and Chinese students concerning their acceptance of and interaction with digital technology for learning, exploratory learning environments and Fractions Lab.

Most of the design of Fractions Lab and its tasks were unchanged from previous studies in the UK and Germany [4], mainly to be able to see its impact on student learning. However, the interface and feedback were translated into Simplified Chinese. Our UK and China studies each involved three primary/elementary schools (in rural, inner-city and suburban contexts). The China schools were all in or around Beijing (in Changping District, Shijingshan District, and Fangshan District). 210 students participated in the UK, and 189 students ( 92 female and 97 male students, aged between 9 and 10 years old, from a total of six classes) participated in China. In each context, the students engaged with Fractions Lab for approximately 45 minutes.

\section{$4 \quad$ Findings and Discussion}

As expected, there were some clear differences between the students in the UK and in China, particularly in relation to institutional practicalities. Some key differences, however, were unanticipated. For example, while in the UK we had employed several 'levels' of feedback [10], including the intentional ambiguity of Socratic questioning, early trials made clear that more work was required to ensure that this approach was appropriate in China. On the other hand, between the two contexts, there were clear observed similarities in the attitudes of the participating students and their teachers. 
For example, in both the UK and China, students were observed to be similarly engaged with Fractions Lab, to make similar errors and hold similar misconceptions, and to have similar reactions to the exploratory nature of the tasks. In addition, teachers in both countries commented that the pre-test was 'too easy' for their students (a claim that was not supported by the test's outcomes, see below). Finally, in both the UK and China, students were observed to enjoy taking advantage of the software's functionality to create the largest possible, although not particularly useful, fraction that the system affords (nine hundred and ninety nine, nine hundred and ninety ninths: 999/999).

In relation to student learning gains, and again consistent with our findings from the UK, pre- and post-test scores were lower than anticipated by the teachers. However, also consistent with the UK, paired samples t-tests showed statistically significant differences between the pre- and post-tests (Table 1), with effect sizes (Cohen's $d$ ) of 0.44 (School A), 0.70 (School B) and 1.00 (School C), all of which together warrant further research.

Table 1. Pre- and post-test scores (each out of a possible 6) of students in the China schools.

\begin{tabular}{lllll}
\hline School & n & Pre-test & Post-test & t-test \\
\hline $\mathrm{A}$ & 64 & $\mathrm{M}=2.53, \mathrm{SD}=1.268$ & $\mathrm{M}=3.11, \mathrm{SD}=1.326$ & $\mathrm{t}(65)=3.470, \mathrm{p}=0.01$ \\
\hline $\mathrm{B}$ & 63 & $\mathrm{M}=2.63, \mathrm{SD}=1.180$ & $\mathrm{M}=3.48, \mathrm{SD}=1.239$ & $\mathrm{t}(64)=4.308, \mathrm{p}<0.05$ \\
\hline $\mathrm{C}$ & 62 & $\mathrm{M}=1.69, \mathrm{SD}=1.207$ & $\mathrm{M}=3.11, \mathrm{SD}=1.575$ & $\mathrm{t}(63)=7.102, \mathrm{p}<0.05$ \\
\hline
\end{tabular}

Regarding student perceptions, we classified the 18 Fractions Lab exploratory tasks into three groups: creation, comparison, and addition and subtraction, and investigated the students' perception of task difficulty (by means of self-reports between each task). However, the data showed no obvious differences in the level of perceived difficulty, suggesting that the support provided by the system helps iron out the differences in what are a range of cognitively demanding tasks (c.f. [11]).

Lastly, although further analysis of interaction data is underway, as in previous research (c.f. [13]), we observe a general tendency for help-avoidance in both countries. Moreover, although we have not observed extensive gaming, perhaps due to novelty effects and the engaging nature of the exploratory tasks, a paired t-test shows significant difference in post-test grades for a group of students who tried to abandon the tasks without spending time interacting $(\mathrm{M}=2.935 \mathrm{SD}=0.245)$ compared with a group who did not attempt to game the system as such $(\mathrm{M}=3.287 \mathrm{SD}=0.109)$; $\mathrm{t}(195)=1.292, \mathrm{P}=0.099$.

\section{Conclusion}

In conclusion, this paper presents work towards a better understanding of cultural differences in relation to educational technology and specifically learning and interaction with exploratory tasks. While at a high-level we are satisfied by the learning performance of students, we are beginning to observe subtle differences in the interaction (more pronounced gaming) but also many similarities that help us develop hypotheses to be answered by more detailed data analysis. This is important because it will help us determine next steps in terms of the design, implementation and integration of the technology in the two contexts. 


\section{Acknowledgements}

This research received funding from the Big Data Centre for Technology-Mediated Education and Beijing Advanced Innovation Center for Future Education. We would like to thank the students and teachers who took part in the studies.

\section{References}

1. A. Ogan, E. Walker, RSJD Baker et al (2012) Collaboration in cognitive tutor use in Latin America: Field study and design recommendations. In: Conference on Human Factors in Computing Systems - Proceedings

2. Z. Miao, D. Reynolds, A. Harris, and M. Jones, 'Comparing performance: a cross-national investigation into the teaching of mathematics in primary classrooms in England and China', Asia Pac. J. Educ., vol. 35, no. 3, pp. 392-403, Jul. 2015.

3. M. Boylan, C. Wolstenholme, B. Maxwell, T. Jay, A. Stevens, and S. Demack, 'Longitudinal evaluation of the mathematics teacher exchange: China-England interim research report', Sheffield Hallam University, DEF-RR559, 2016.

4. N. Rummel et al., 'Combining Exploratory Learning with Structured Practice to Foster Conceptual and Procedural Fractions Knowledge', in ICLS, Singapore, 2016.

5. A. Hansen, M. Mavrikis, and E. Geraniou, 'Supporting teachers' technological pedagogical content knowledge of fractions through co-designing a virtual manipulative', J. Math. Teach. Educ., vol. 19, no. 2-3, pp. 205-226, Apr. 2016.

6. C. Mazziotti et al., 'Robust Student Knowledge: Adapting to Individual Student Needs as They Explore the Concepts and Practice the Procedures of Fractions (workshop paper)', presented at AIED, Madrid, 2015.

7. B. Grawemeyer, M. Mavrikis, W. Holmes, S. Gutiérrez-Santos, M. Wiedmann, and N. Rummel, 'Affective learning: improving engagement and enhancing learning with affectaware feedback', User Model. User-Adapt. Interact., pp. 1-40, 2017.

8. B. Rittle-Johnson and M. W. Alibali, 'Conceptual and procedural knowledge of mathematics: Does one lead to the other?', J. Educ. Psychol., vol. 91, pp. 175-189, 1999.

9. M. Wong and D. Evans, 'Students' conceptual understanding of equivalent fractions', in Mathematics: Essential Research, Essential Practice, 2007, vol. 2, pp. 824-833.

10. W. Holmes, M. Mavrikis, A. Hansen, and B. Grawemeyer, 'Purpose and Level of Feedback in an Exploratory Learning Environment for Fractions', in Artificial Intelligence in Education, vol. 9112, C. Conati, N. Heffernan, A. Mitrovic, and M. F. Verdejo, Eds. Cham: Springer International Publishing, 2015, pp. 620-623.

11. B. Grawemeyer, M. Mavrikis, C. Mazziotti, A. van Leeuwen, N. Rummel (to appear) The impact of affect-aware support on learning tasks that differ in their cognitive demands. Artificial Intelligence in Education proceedings

12. Roll, R. S. J. d Baker, V. Aleven, and K. R. Koedinger, "On the Benefits of Seeking (and Avoiding) Help in Online Problem-Solving Environments," Journal of the Learning Sciences, vol. 23, no. 4, pp. 537-560, Oct. 2014 